\title{
Free Code Sharing Web Platform with Intelligent Developer Suggestion Tool
}

\author{
Ashish A Gaikwad, Deepali R Vora
}

\begin{abstract}
Due to increase in demands for websites, faster deployment requirements and complex functionality requirements, developers face the need for a centralized code repository system to store repetitive codes and a suggestion tool to help them in solving the errors and warnings they face during development. The proposed system provides a centralized database for storing repetitive code segments, errors, warnings and solutions and also a code suggestion mechanism to provide suggestions to the developer during development. The system includes a code collection system to help the user to add the codes, errors and solutions to the centralized database and an analyzer system to fetch the web-page data, errors and warnings to analyze and provide appropriate suggestions to the developer. The proposed system focuses to reduce work and development time of developers by providing code suggestions during development and also facilitate effective code reusability.
\end{abstract}

Keywords: Intelligent System, Browser extension, Analyzer system, Suggestion Tool

\section{INTRODUCTION}

Currently the developer's works includes saving reusable code segments and modules to private repositories or excel sheets of the organization in which the developer is working. The developer also has to search the internet for solutions to the errors found during development and find code segments of unknown functionalities. This work is time consuming and uses expensive development time. This reduces the efficiency and productivity of an organization.

Also, the solutions to the developer requirements are on different and distributed web platforms. So there is no centralized system for storage of solutions to the errors and repetitive code segments. The code needs to be searched from different sites and blogs. The developer loses his data once he changes the organization. So in order to preserve the code repository and integrate the reusable code data of developers of different organizations

It is suggested to create and use a centralized web application and repository. It is suggested to use browser extension or task runners to fetch errors and warnings and

Revised Manuscript Received on December 30, 2019.

* Correspondence Author

Mr. Ashish Arjun Gaikwad, Department of Information Technology, Vidyalankar Institute of Technology, Mumbai, India. Email: ashishagaikwad@gmail.com

Dr. Deepali Vora, Department of Information Technology, Vidyalankar Institute of Technology, Mumbai, India. Email: deepali.vora@vit.edu.in

(C) The Authors. Published by Blue Eyes Intelligence Engineering and Sciences Publication (BEIESP). This is an open access article under the CC BY-NC-ND license (http://creativecommons.org/licenses/by-nc-nd/4.0/) provide them to a system which can analyze them and suggest proper and relevant suggestions to the developers to resolve the errors and warnings during development time and testing.

To resolve the problems of developers who need support to resolve errors and warnings during development time it is suggested to create a browser extension which could scan the console and web pages to pick up the errors and warnings, send them to intelligent system for parsing and fetch the relevant solutions and suggest it to the developer during development time. This reduces the search time required by the developer and increases efficiency and productivity.

The browser extension can further determine the language and the framework or CMS of the web page during initial page load and speed up the process of suggesting relevant solutions for errors and requests for code fragments with the help of the intelligent system. The extension does the scan at regular time intervals and on initial page load. The extension also provides an interface for searching code segments and adding new code segments from the extension.

This will make it simple to gather repetitive code segments. It can also give SEO suggestion and vulnerability warnings to the web page user. Further it can suggest repetitive code segments like Google analytics code and Shiv and Shim codes to the user. For the logged in user the system can analyze his previous searches and suggest relevant code segments. The extension can also provide personally saved snippets or templates to the user.

The extensions required for assessment needs to be developed for different browsers to improve the application usage. The solutions to the errors and the reusable code segments are saved as a blog where other users can comment and up-vote the answers. The intelligent system parses the requests of data and errors and provides the relevant suggestion after searching the parsed keywords. The intelligence system uses the database for storing and accessing the data. The database system needs to be periodically indexed and optimized to ensure best performance of the system. There is a provision of periodic backup of the database to avoid data loss due to hacking. Multiple Answers can be recorded for a single issue description due to which developers will have a high probability of finding suitable answers. While users can also up vote their accepted solution to highlight the best solution. The solution with the most votes is considered to be the best solution. While the extensions can provide necessary functions, there is a need to check the vulnerabilities of the extensions and provide necessary security features to minimize them. 


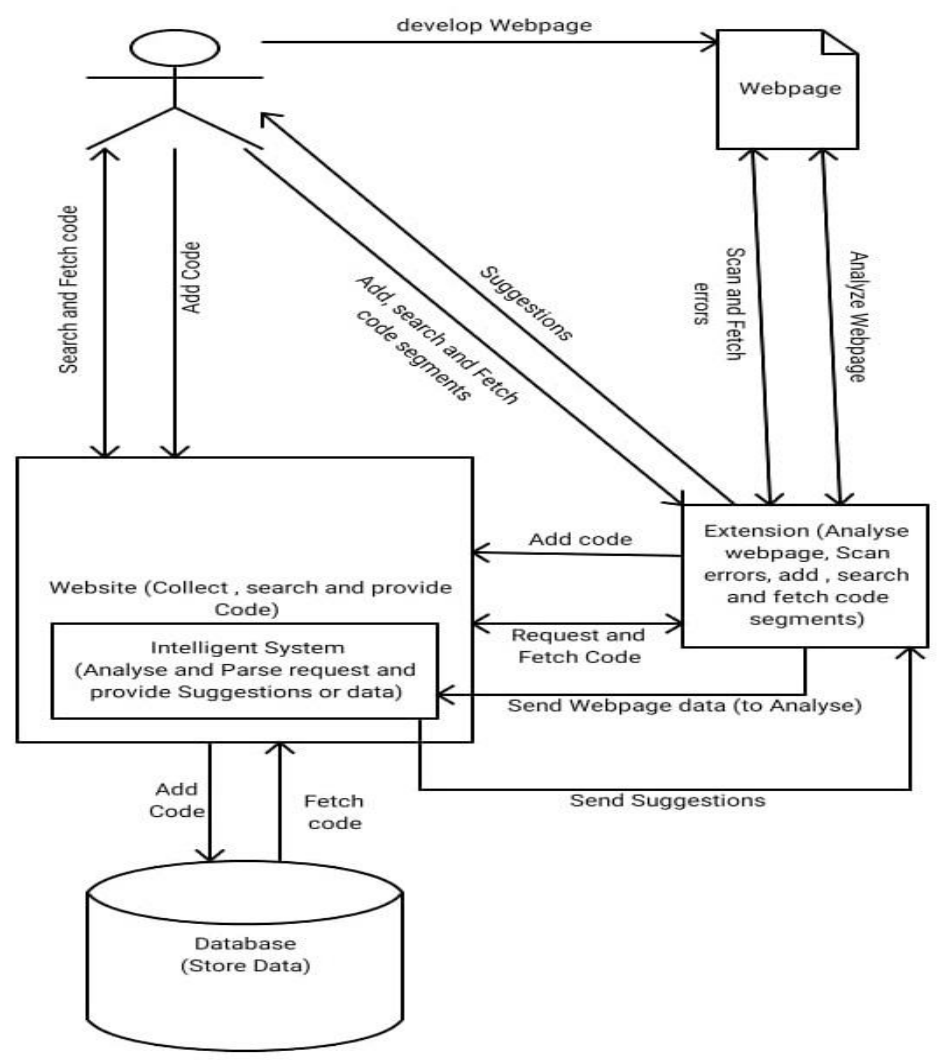

Fig. 1.Basic System Architecture

\section{LITERATURE REVIEW}

Donghui Wang describes ways to enhance the parser to manage complex mathematical and logical expressions. This new ways describe a technique with shunting yard style algorithm and other techniques after the original canonical LALR providing two level parsing approach [1].

David A. Botwe et al describe web application as a program which runs on the browser and can be accessed from different locations by a network. There are different technologies used for the design and development of the websites. They describe a comparison of web application development technologies like PHP, ASP.NET and JSP using some parameters like implementation cost, browser compatibility, response time, Operating system and mobile platform compatibility [2].

Dr. N. Yuvaraj, S. Gowdham etal explain us about method to do on page optimization and improve the web page ranking in the search engine listings. It focuses on optimizing the page content by focusing on various keywords without using any keyword stuffing [3].

Frolin S. Ocariza, Jr. etal describe about the errors involved in the Javascript implementation and their types. Javascript is a client scripting language used to make the application. They also state the reliability of coding using JavaScript in application development and help us to find the root causes of the JavaScript errors [4].

Nalaka R Dissanayake et al describe AJAX as a powerful scripting approach in developing Rich Internet Applications however due to some complexities involved it is difficult to implement Rapid Application development which can be resolved by providing standard framework and methods. In their paper we study the different practices which can help us to achieve Rapid application development using AJAX to create rich internet applications [5].

Arjun Guha et al describe the security features that can be implemented in browser extension to prevent malicious attacks from the hackers and viruses [6].

Dr.-Ing. Mario Heiderich Alex Inführ et al describe different ways to protect web browser feom hacker attacks and protect websites data [7].

Majid Khan and M. N. A. Khan describe various methods to optimize relational databases and sql queries to ensure good performance and speed [8].

Bindu Sharma and Mahesh Singh describe different methods to optimize database using time and cost analysis using neural networks [9].

Barth, A. P. Felt and P. Saxethem explain their study about the vulnerabilities posed by the browser extensions and the different methods to protect the web pages from those vulnerabilities. Different browsers have different methods of developing extensions and have different vulnerabilities. A developer has to understand these vulnerabilities and remove them during the making of the extension [10].

Bruch et al explain and describe different ways to improve the code completion system. The code completion system works on fetching of code snippets based on keywords [11].

\section{PROPOSED SYSTEM}

In this system, our goal is to design a system which can provide centralized repository to developers for saving their reusable codes and solutions to the common errors and also provide development time suggestion to the errors reducing their time and effort to search the solutions from different websites.

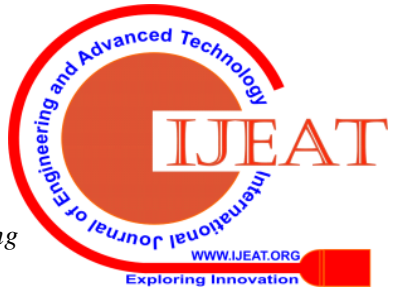


This system can also suggest the developer's relevant code segments by analyzing the user preferences and web page assessment. Further we can also provide suggestions to the developers for optimizing the web pages by analyzing their webpage data. Our proposed system consists of four parts consisting of website, Analyzer system, database and intelligent system.

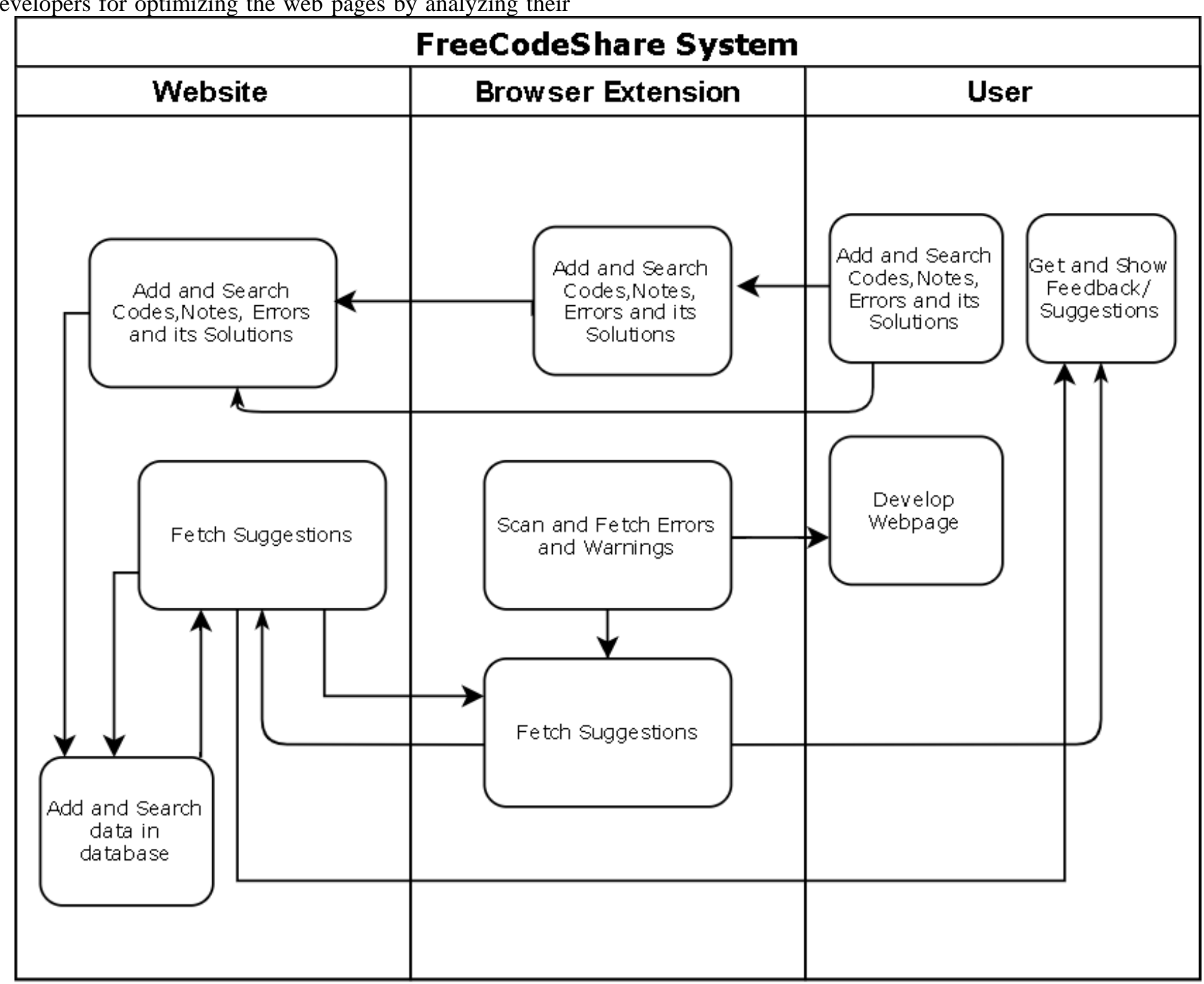

Fig. 2.FreeCodeShare System Flowchart

\section{A. Code Gathering, Search and Error detection (Website and Browser Extension)}

The website acts as an interface to the developers for storing their reusable code and also search and fetch the code and error solutions added by other developers. The website system also allows the developers to access their saved codes at a common place from the repository at any time.

It helps the developer to add the reusable codes with a proper classification according to its language, frameworks and CMS used in the development. The system also analyses the users search records and shows him relevant code segments accordingly.

The Analyzer system consists of the extension which is used to access the web page and console of the developer and provide them the solutions to the errors found on the web page and the console. It can also provide the relevant code suggestions to the developer to increase the speed of the development and make effective use of reusable code segments or snippets and templates. The database will act as a centralized repository for storing and accessing the data. The database optimization like indexing and other query optimization techniques will be done to improve the data access speed of the database. Frequent Database backup will be provided at regular intervals to fight with issues like data loss and hacking attacks 


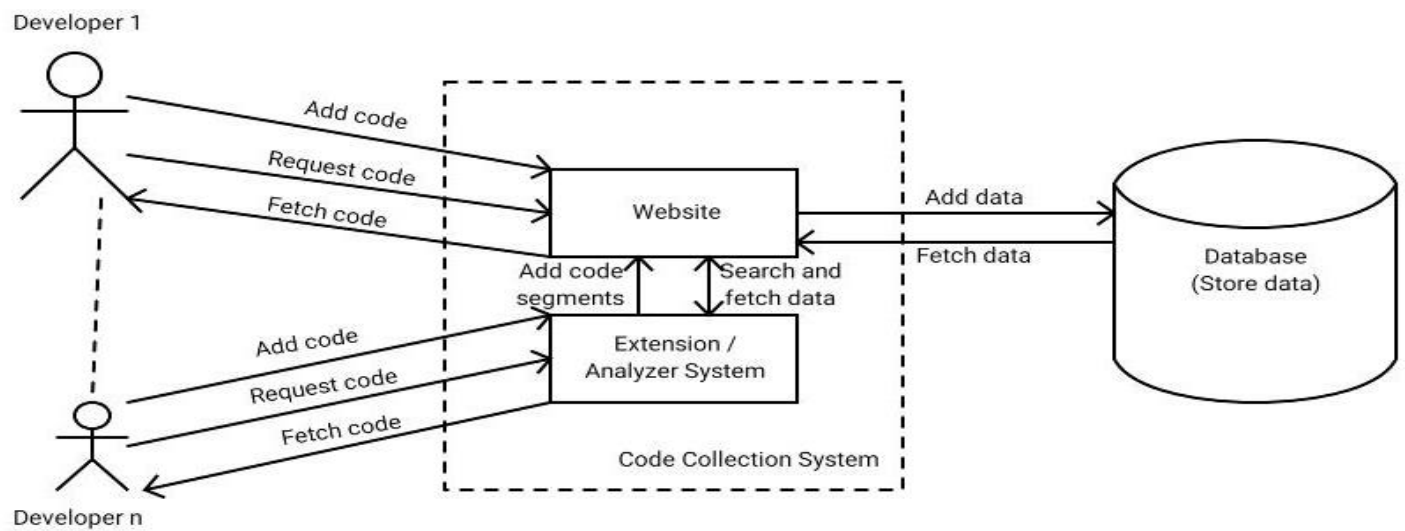

Fig. 3.Code Gathering

\section{B. Assessment and Suggestion System (Intelligent System)}

In this step the extension sends the web page data to intelligent system to analyses the users system to predetermine the language and the frameworks and CMS used the user in the initial loading of the web page. Browser extension is used to do the assessment of the web page of the user.

It scans the web page on intervals to find out errors and if it finds out the errors then it passes the errors to the intelligent system for analysis and retrieves and provides the solution to the user.

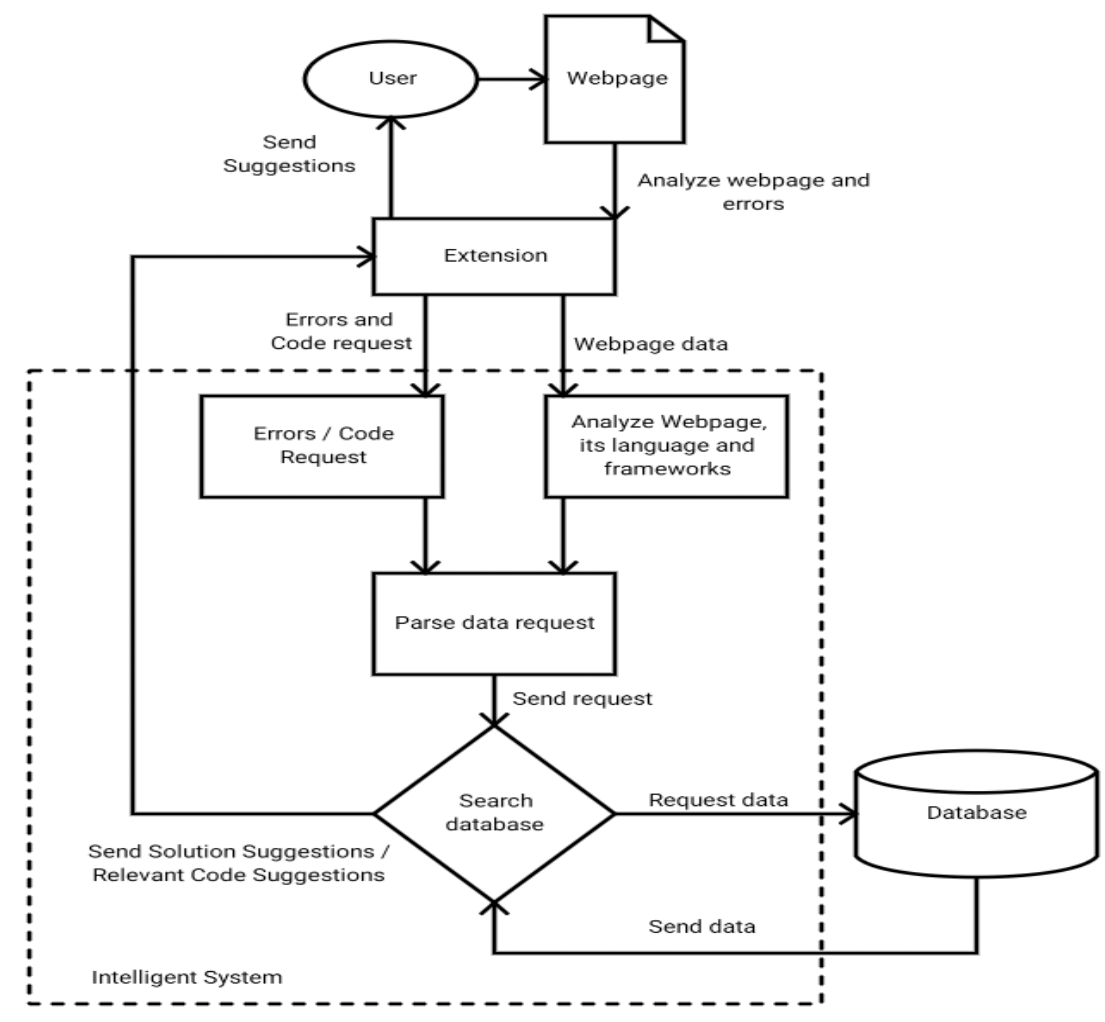

Fig. 4.Intelligent System

It has following functions:

- Determine programming languages:Analyze the web page by extension during the initial load of the web page to find out the language and frameworks and CMS used.

- Find Errors: Check the web page and the console for the errors and recheck at regular intervals.

- Send Error and Provide Suggestions: If any errors are found then the extension sends the errors to the intelligent system to parse the error and provide the suggestion back to the developer.

- Provide Specific Search: If the user wants to search for a specific data he can use the extension to input the query and get the suggestions or code segments. 
Provide Suggestions on Web Page Analysis: The extension can also analyze the web page to find the relevant predefined search tags and provide it to intelligent system to give relevant suggestions to the user.

\section{Algorithms}

The Analyzer System implemented by using the browser extension helps to analyze code, add data, search data and fetch suggestions for the user. It consists of following steps:

- Add Codes, Notes, Errors and Solutions from the users.

- Search Codes, Notes, Errors and solutions required by the user and provide them suggestions and fetched code data.

- Analyze the webpage of the developer and scan the web browser console.

- Send the data to the intelligent system in Website for analysis.

- $\quad$ Fetch and show the suggestion data to the user.

The intelligent system helps to parse request, analyze data and provide suggestions to the user. It works in following steps:

- Parse User Request.

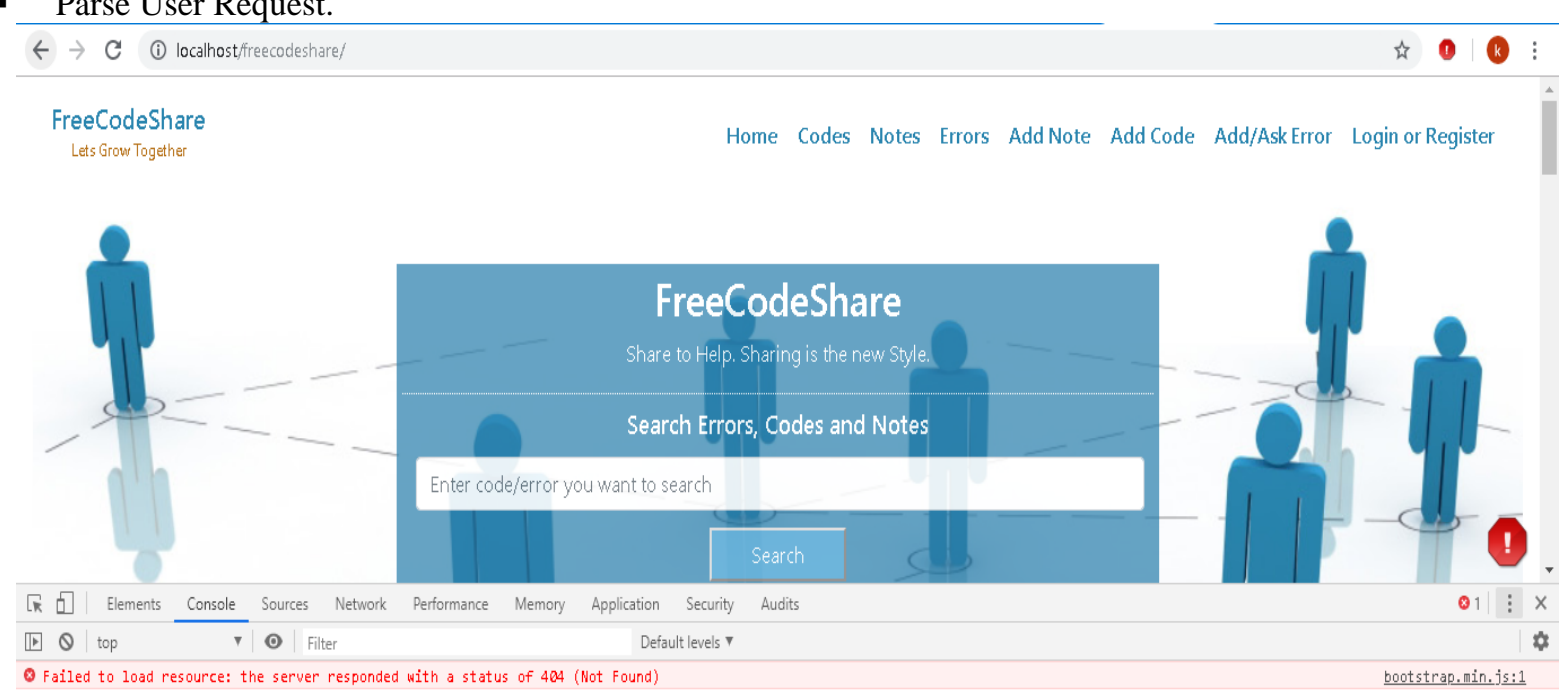

Fig. 5.Sample Error Detection

The browser extension is created using HTML, CSS, Javascript and JQuery. The codes segments are directly shown using the modal boxes. The website provides the platform where users can check the latest codes added in their languages, frameworks and CMS used.There are different sections where users can check their own added codes, edit them and delete them.

The users are notified when someone comments on their added codes, notes and solutions. Various security factors are checked before getting the input from the user to prevent against malicious attacks. The system used a MD5 hash algorithm for encrypting password data and base64 and SHA algorithm for encrypting other sensitive data.

The intelligent system in the website fetches the relevant code segments present in the database and provides them to the user or developer. It helps the developers to get development time error and code suggestions without having
- Search relevant data from the database.

- Shortlist the search results to find most relevant data as per the request.

- Provide the suggestions to the user.

- Fetch user feedback and save the user preferences in the database for helping future searches.

\section{RESULTS AND DISCUSSION}

The concept is implemented with the website www.freecodeshare.in and the freecodeshare Analyzer browser extension which can be downloaded from the website. In this website the developers can store their reusable codes, errors and solutions to the errors. The user can also do a normal search on the website to find his entered search keywords.

The search results are fetched from the codes data, notes data and the errors and solutions data. Errors are categorized according to their error code, language and scripts used. The developers or users can also add comments to the code, notes and solutions.The website is implemented in PHP language using a codeigniter framework and various jquery and javascript libraries. 


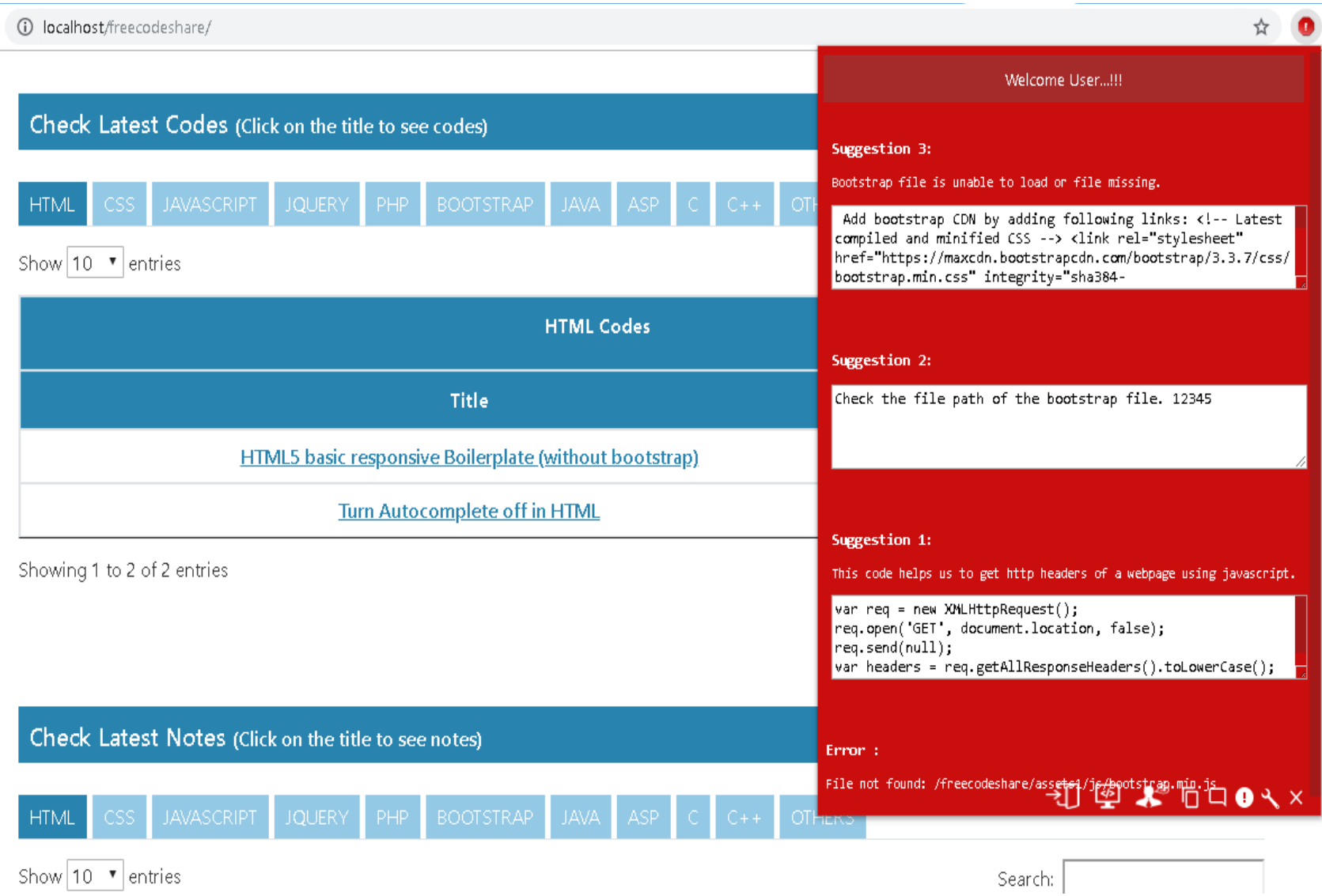

Fig. 6.Suggestions in Browser Extension (Analyzer System)

The developer has to decide which suggestion can help him resolve his problem better. Suggestions can be found out by the intelligent system by analyzing the code segments, notes segments and the solutions for the errors stored in the centralized database.

\section{CONCLUSION}

The problem of code reusability while changing different organizations and the need of solutions or suggestions for errors and warnings during development time can be resolved by the proposed system. The proposed system can improve the development efficiency by reducing the time and effort requirements of the developer and will also help to make a centralized architecture for storing of the reusable code segments data and help in providing easy access to code segments. The system will also help the developers to retain their reusable code segments irrespective of their organization and thus stop the code loss and improve higher interaction between developers across the globe.

The need of more relevant solutions can be completed by up voting and down voting a solution by developers. The developers can add and search the code segments from the website and can also provide a new solution which can help the developer community to get multiple solutions for the required data. Since the browser extension or the analyzer system can also analyze the code of the web page of the developer and suggest him relevant code segments then it can also improve the development speed of the developers. If this system is properly implemented, improved and utilized then it will prove a boon for the developers to make projects faster and effectively facilitate reuse of code segments from all over the world.

\section{REFERENCES}

1. Donghui Wang,"A Practical parser with combined parsing techniques", International JournalofSoftware Engineering \& Applications (IJSEA), Vol.6, No.4, July 2015.

2. David A. Botwe, Joseph G. Davis,"A Comparative Study of Web Development Technologies Using Open Source and Proprietary Software", International Journal of Computer Science and Mobile Computing, IJCSMC, Vol. 4, Issue. 2, pp.154-165, 2015.

3. Dr. N. Yuva `aj, S. Gowdham, V.M. Dinesh Kumar and S. ohammed Aslam Batcha, "On-page search engine optimization analysis",N.Yuvaraj etal,/(IJCSIT) International Journal of Computer Science and Information Technologies, Vol. 8 (2), 2017.

4. Frolin S. Ocariza, Jr., Karthik Pattabiraman, Benjamin Zorn, "JavaScript Errors in the Wild: An Empirical Study", University ofBritish Columbia,Microsoft Research, Redmond, WA, USA.

5. Nalaka R Dissanayake, Kapila Asanga Dias ,"Best Practices for Rapid Application Development of AJAX based rich internet applications", Conference Paper December 2014.

6. Arjun Guha, Matthew Fredrikson, Benjamin Livshits, Nikhil Swamy, "Verified Security for Browser Extensions", IEEE Symposium on Security and Privacy, 2011.

7. Dr.-Ing. Mario Heiderich Alex Inführ, MSc. Fabian Fäßle BSc.Nikolai Krein, MSc. MasatoKinugawa Tsang-Chi "Filedescriptor" Hong, BSc.Dario Weißer, BSc. Dr. Paulina Pustułka, "Cure53 Browser Security White Paper", Cure53, Berlin, 2017.

8. Majid Khan, M N A Khan, "Exploring Query Optimization Techniques in Relational Databases", International Journal ofDatabase Theoryand Application,Vol. 6, No. 3, June, 2013.

9. Bindu Sharma, Mahesh Singh, "Performance Tuning in Database Management System based on Analysis of Combination of Time and Cost Parameter through Neural Network Learning”, International Journal of Computer Applications (0975 - 8887), Volume 96-No.1, June 2014. 
10. A. Barth, A. P. Felt, and P. Saxena. Protecting browsers from extension vulnerabilities. In NDSS, 2010

11. Bruch, Marcel; Monperrus, Martin; Mezini, Mira (2009). "Learning from examples to improve code completion systems": (213-222). doi: $\underline{10.1145 / 1595696.1595728}$

\section{AUTHORS PROFILE.}

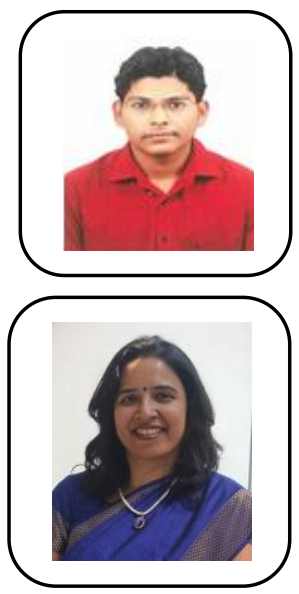

Mr. Ashish Arjun Gaikwad, is an M.E Student in Department of Information Technology Vidyalankar Institute of Technology, Mumbai, India. He has interest in Web Application, data science and artificial Intelligence.

Dr. Deepali Vora, is a professor in Vidyalankar Institute of Technology Mumbai. She received her $\mathrm{PhD}$ in Computer Science from Amity University, Mumbai in 2019. Her research interests lie in the machine learning and data science. 